\author{
I.N. Parasidis
}

University of Thessaly, Gaiopolis, Greece

(E-mail: gparasidis@gmail.com)

\title{
Extension method for a class of loaded differential equations with nonlocal integral boundary conditions
}

In this paper we investigate a class of loaded ordinary differential equations with nonlocal integral boundary conditions in terms of an abstract operator equation

$$
\begin{gathered}
B u=A^{2} u-q \Psi(u)=f, \quad f \in Y, \\
D(B)=\left\{u \in D\left(A^{2}\right): \Phi(u)=N F(A u), \quad \Phi(A u)=P F(A u)\right\} .
\end{gathered}
$$

A loaded part and nonlocal integral boundary conditions of these equations are described using functional vectors $\Psi(u)$ and $F(A u)$, respectively. Such equations follow from Extension Theory of linear operators. The necessary and sufficient solvability conditions of these equations are given by the determinant of some matrix. In the case when this determinant is nonzero, a direct method for exact solution of this class of loaded differential equations is proposed. If some problem can be reduced to the type of equation under consideration, then it can be easily solved using the extension method. This method, for $q=\overrightarrow{0}$, also gives the necessary and sufficient solvability conditions and the exact solution of a class of ordinary differential equations with nonlocal integral boundary conditions in terms of an abstract operator equation $B u=A^{2} u=f, \quad D(B)=\left\{u \in D\left(A^{2}\right): \Phi(u)=N F(A u), \quad \Phi(A u)=P F(A u)\right\}, \quad f \in Y$.

Keywords: loaded ordinary differential equations, differential equations, nonlocal integral boundary conditions, injective and correct operators, exact solutions.

\section{Introduction}

In recent years the theory of loaded functional and differential equations (or equations with aftereffect) has been advanced. These equations describe the problems of optimal control, such as: longstanding prediction method and regulation of the layer of soil water and ground moisture, the problems of underground fluid and gas dynamics, mathematical biology, ecology and economics [1-5]. The stationary one-speed transport equation is an important example of loaded differential equations [6]. A methodical study on the theory of boundary value problems for loaded functional and differential equations and their applications has been conducted by Nakhushev [4]. An equation is called loaded if it contains the solution function on a manifold with dimension less than the dimension of domain of this function [4]. For example an ordinary loaded differential equation is represented by

$$
d y / d x=f(x, y)+h\left(y\left(x_{j}\right)\right), \quad x \in[0,1], x_{j} \in[0,1],
$$

where $x_{j}$ are fixed points. Boundary value problems consisting of general boundary conditions and the so-called differential boundary equations (loaded differential equations) have been investigated by many researchers in the last century, see, for example, the survey paper by Krall [7] and the references cited in it. Big interest represent the theory of loaded equations in Pure and Applied Mathematics [8-15]. Usually boundary value problems for the loaded ordinary differential equations with integral boundary conditions are investigated by numerical methods [16-19]. Here, the necessary and sufficient solvability conditions of the abstract operator equations (1) and their exact solutions by Extension Method are obtained in closed form. This formalism is applied to solve the ordinary loaded differential equations with integral boundary conditions, when $q, \Phi, \Psi, F$ are vectors, $N, P$ matrices, $A$ is an ordinary differential operator, $\Psi(u)$ is a loaded part of equations and $F(A u)$ defines the integral boundary conditions. Such problems arise naturally from Oinarov extensions of linear operators [20, 21] in Banach space which are not restrictions of a maximal operator, unlike the classical M. Krein, J. Von. Neuman extensions [22, 23] in Hilbert space and Otelbaev, Kokebaev, Shynybekov extensions [24] in Banach space. Extension Method uses a symple correct restiction $\widehat{A}$ of a maximal operator $A$ defined by $\widehat{A} u=A u, \quad D(\widehat{A})=\{u \in D(A): \Phi(u)=\mathbf{0}\}$, to solve complex problems of type (1). This method was applied in [25] to simpler abstract operator equations $B u=A u-q \Psi(u)=f, \quad D(B)=\{u \in D(A): \Phi(u)=N F(A u)\}, \quad f \in Y$ for the study of ordinary loaded differential and loaded integro-differential equations with integral boundary conditions and their exact solutions in closed form. There are many problems of type (1). The loaded differential operator [8] 


$$
\begin{aligned}
& L u(x)=u^{\prime \prime}(x)+a(x) u\left(\frac{1}{4}\right)+b(x) u\left(\frac{1}{2}\right)+u(1) ; \\
& u(0)=\int_{0}^{1} \gamma(x) u(x) d x, \quad u^{\prime}(1)=\int_{0}^{1} \nu(x) u(x) d x,
\end{aligned}
$$

is an operator of type (1) if we take $A u=u^{\prime}(x), q_{1}=-a(x), q_{2}=-b(x), q_{3}=-1, \Psi_{1}(u)=u(1 / 4)$, $\Psi_{2}(u)=u(1 / 2), \quad \Psi_{3}(u)=u(1), \Phi_{1}(u)=u(1), \Phi_{2}(u)=u^{\prime}(1), \int_{0}^{1} \gamma(x) d x=\int_{0}^{1} \nu(x) d x=0, \quad \widehat{A} u=A u$, $D(\widehat{A})=\{u(x) \in D(A): u(1)=0\}$. Then $\widehat{A}^{-1} f(x)=\int_{1}^{x} f(x) d x$ and the boundary conditions of $(2)$ are represented as

$$
u(1)=\int_{0}^{1}\left[1-\int_{0}^{x} \gamma(s) d s\right] u^{\prime}(x) d x, \quad u^{\prime}(1)=-\int_{0}^{1} \int_{0}^{x} \nu(s) d s u^{\prime}(x) d x .
$$

So $N=1$,

$F_{1}(A u)=\int_{0}^{1}\left[1-\int_{0}^{x} \gamma(s) d s\right] u^{\prime}(x) d x, \quad P=-1, \quad F_{2}(A u)=\int_{0}^{1} \int_{0}^{x} \nu(s) d s u^{\prime}(x) d x$ and Extension Method can be applied.

The loaded differential operator [8]

$$
\begin{gathered}
L v(x)=v^{\prime \prime}(x)+\gamma(x) v^{\prime}(0)+\nu(x) v(1) ; \\
v(0)=0, \quad v^{\prime}(1)=\int_{0}^{1} v(x) d x
\end{gathered}
$$

is also an operator of type (1) if we take the operators $A, \widehat{A}$, the vectors $\Phi_{1}, \Phi_{2}$ as in $(2)$ and $q_{1}=-\gamma(x)$, $q_{2}=-\nu(x), \Psi_{1}(v)=v^{\prime}(0), \Psi_{2}(v)=v(1)$. Note that the boundary conditions of (3) are represented as $v(1)=\int_{0}^{1} v^{\prime}(x) d x, v^{\prime}(1)=\int_{0}^{1}(1-x) v^{\prime}(x) d x$. So $N=P=1, F_{1}(A v)=\int_{0}^{1} v^{\prime}(x) d x, F_{2}(A v)=\int_{0}^{1}(1-$ $-x) v^{\prime}(x) d x$, and Extension Method can be applied. The technique of this method is simple to use and can be easily incorporated to any Computer Algebra System (CAS). The paper is organized as follows. First we recall some basic terminology and notation about operators. Then we prove the main general results and give an example of boundary value problem with ordinary loaded differential equation and nonlocal integral boundary conditions which show the usefulness of our results.

\section{Terminology and notation}

Let $X, Y$ be a complex Banach spaces and $X^{*}$ is the adjoint space of $X$, i.e. the set of all complex-valued linear and bounded functionals on $X$. We denote by $f(x)$ the value of functional $f \in X^{*}$ on $x \in X$. We write $D(A)$ and $R(A)$ for the domain and the range of the operator $A$, respectively. An operator $A_{2}$ is said to be an extension of an operator $A_{1}$, or $A_{1}$ is said to be a restriction of $A_{2}$, in symbol $A_{1} \subset A_{2}$, if $D\left(A_{2}\right) \supseteq D\left(A_{1}\right)$ and $A_{1} x=A_{2} x$, for all $x \in D\left(A_{1}\right)$. An operator $A$ is called maximal if $R(A)=Y$ and $\operatorname{ker} A \neq\{0\}$. An operator $\widehat{A}: X \rightarrow Y$ is said to be correct if $R(\widehat{A})=Y$ and the inverse $\widehat{A}^{-1}$ exists and is continuous on $Y$. An operator $\widehat{A}$ is called a correct restriction of the maximal operator $A$ if it is a correct operator and $\widehat{A} \subset A$. An operator $A: X \rightarrow Y$ is said to be injective if for all $u_{1}, u_{2} \in D(A)$ such that $A u_{1}=A u_{2}$, follows that $u_{1}=u_{2}$. Remind that a linear operator $A$ is injective if and only if $\operatorname{ker} A=\{0\}$. If $\Psi_{i} \in X^{*}, i=1, \ldots, n$, then we denote by $\Psi=\operatorname{col}\left(\Psi_{1}, \ldots, \Psi_{n}\right)$ and $\Psi(x)=\operatorname{col}\left(\Psi_{1}(x), \ldots, \Psi_{n}(x)\right)$. Let $g=\left(g_{1}, \ldots, g_{n}\right)$ be a vector of $X^{n}$. We will denote by $\Psi(g)$ the $n \times n$ matrix whose $i, j$-th entry $\Psi_{i}\left(g_{j}\right)$ is the value of functional $\Psi_{i}$ on element $g_{j}$. Note that $\Psi(g C)=\Psi(g) C$, where $C$ is a $n \times k$ constant matrix. We will also denote by $\mathbf{0}_{l n}$ the zero $l \times n$ matrix and by $I_{n}$ the identity $n \times n$ matrix. By $\overrightarrow{0}$ we will denote the zero column vector.

Let $A: X \stackrel{\text { on }}{\rightarrow} Y$ be an ordinary $m$ order differential operator

$$
A u(x)=\alpha_{0} u^{(m)}(x)+\alpha_{1} u^{(m-1)}(x)+\ldots+\alpha_{m} u(x), \quad \alpha_{i} \in \mathbb{R}, \alpha_{0} \neq 0
$$

and $X, Y$ be the Banach spaces. Usually $X=Y=C[a, b]$ or $X=Y=L_{p}(a, b), p \geq 1$. Everywhere below we denote by

$$
X_{A}^{m}=\left(D(A),\|\cdot\|_{X_{A}^{m}}\right)
$$

the Banach space $m$ times differentiable functions with norm

$$
\|u(x)\|_{X_{A}^{m}}=\sum_{i=0}^{m}\left\|u^{(i)}(x)\right\|_{X}
$$


It is a well-known fact that the operator $\widehat{A}: C[a, b] \stackrel{o n}{\rightarrow} C[a, b]$ defined by

$$
\begin{gathered}
\widehat{A} u(x)=A u(x)=f, \\
D(\widehat{A})=\left\{u(x) \in C^{m}[a, b]: u\left(x_{0}\right)=u^{\prime}\left(x_{0}\right)=\ldots=u^{(m-1)}\left(x_{0}\right)=0\right\}, \quad x_{0} \in[a, b],
\end{gathered}
$$

is a correct restriction of $A$ and the unique solution of (5) for $\alpha_{0}=1, \alpha_{1}=\ldots=\alpha_{m}=0$ is

$$
u(x)=\widehat{A}^{-1} f(x)=\frac{1}{(m-1) !} \int_{x_{0}}^{x}(x-t)^{m-1} f(t) d t, \quad f(x) \in C[a, b] .
$$

Lemma 1. Let $X, Y$ be complex Banach spaces, $A: X \stackrel{\text { on }}{\rightarrow} Y$ an operator defined by (4) with finite dimensional kernel $\mathbf{z}=\left(z_{1}, \ldots, z_{m}\right)$ which is a basis of ker $A$. Suppose also that the components of a functional vector $\Phi=\left(\Phi_{1}, \ldots, \Phi_{m}\right) \quad$ belong to $\left[X_{A}^{m}\right]^{*}$, a set $\Phi_{1}, \ldots, \Phi_{m}$ is biorthogonal to $z_{1}, \ldots, z_{m}$, i.e. $\Phi(\mathbf{z})=I_{m}$ and $\widehat{A}$ is a correct restriction of $A$ defined by

$$
\widehat{A} \subset A, \quad D(\widehat{A})=\{u \in D(A): \Phi(u)=0\} .
$$

Then the operator $\widehat{A}^{2}$ is correct and defined by

$$
\widehat{A}^{2} \subset A^{2}, \quad D\left(\widehat{A}^{2}\right)=\left\{u \in D\left(A^{2}\right): \Phi(u)=0, \quad \Phi(A u)=0\right\} .
$$

Proof. By definition since (7) we get

$$
\begin{gathered}
D\left(\widehat{A}^{2}\right)=\{u \in D(\widehat{A}): \widehat{A} u \in D(\widehat{A})\}= \\
=\{u \in D(A): \Phi(u)=0, \widehat{A} u \in D(A), \Phi(\widehat{A} u)=0\}= \\
=\{u \in D(A): \Phi(u)=0, A u \in D(A), \Phi(A u)=0\}= \\
=\left\{u \in D\left(A^{2}\right): \Phi(u)=0, \quad \Phi(A u)=0\right\} .
\end{gathered}
$$

Then $\widehat{A}^{2} \subset A^{2}$. Finally the operator $\widehat{A}^{2}$ is correct as superposition of two correct operators.

Remark 2. If the operator $\widehat{A}$ is defined by (5) then

$$
\begin{gathered}
\widehat{A}^{2} u(x)=\alpha_{0} u^{(2 m)}(x)+\alpha_{1} u^{(2 m-1)}(x)+\ldots+\alpha_{2 m} u(x) ; \\
D\left(\widehat{A}^{2}\right)=\left\{u(x) \in C^{2 m}[a, b]: u\left(x_{0}\right)=u^{\prime}\left(x_{0}\right)=\ldots=u^{(2 m-1)}\left(x_{0}\right)=0\right\}
\end{gathered}
$$

Theorem 3. Let the spaces $X, Y$, the operators $A, \widehat{A}$ and a vector $\Phi$ be defined as in Lemma 1 , and the components of the functional vectors $\Psi=\operatorname{col}\left(\Psi_{1}, \ldots, \Psi_{l}\right)$ and $F=\operatorname{col}\left(F_{1}, \ldots, F_{n}\right)$ belong to $\left[X_{A}^{m}\right]^{*}$ and $Y^{*}$, respectively. Suppose also that the components of the vector $q=\left(q_{1}, \ldots, q_{l}\right)$ are linearly independent on $Y$ and $N, P$ are the $m \times n$ constant matrices. Then:

(i) The operator $B$ defined by

$$
\begin{gathered}
B u=A^{2} u-q \Psi(u)=f, \quad f \in Y, \\
D(B)=\left\{u \in D\left(A^{2}\right): \Phi(u)=N F(A u), \quad \Phi(A u)=P F(A u)\right\},
\end{gathered}
$$

is injective if and only if

$$
\operatorname{det} L=\operatorname{det}\left(\begin{array}{cc}
I_{l}-\Psi\left(\widehat{A}^{-2} q\right) & -\Psi\left(\widehat{A}^{-1} \mathbf{z} P+\mathbf{z} N\right) \\
-F\left(\widehat{A}^{-1} q\right) & I_{n}-F(\mathbf{z}) P
\end{array}\right) \neq 0 .
$$

(ii) If $B$ is injective, then $B$ is correct and for all $f \in Y$ the unique solution of (8) is given by

$$
u=B^{-1} f=\widehat{A}^{-2} f+\left(\widehat{A}^{-2} q, \widehat{A}^{-1} \mathbf{z} P+\mathbf{z} N\right) L^{-1}\left(\begin{array}{c}
\Psi\left(\widehat{A}^{-2} f\right) \\
F\left(\widehat{A}^{-1} f\right)
\end{array}\right) .
$$

Proof (i). From boundary conditions (8), since $\Phi(\mathbf{z})=I_{m}$, we obtain

$$
\Phi(u-\mathbf{z} N F(A u))=0, \quad \Phi(A u-\mathbf{z} P F(A u))=0 .
$$


From (11), taking into account (7), we get $u-\mathbf{z} N F(A u) \in D(\widehat{A}), \quad A u-\mathbf{z} P F(A u) \in D(\widehat{A})$. Further, using these relations, the correctness of $\widehat{A}$ and $\mathbf{z} \in[\operatorname{ker} A]^{m}$, for every $u \in D(B)$ from (8) we obtain

$$
\begin{gathered}
B u=A(A u-\mathbf{z} P F(A u))-q \Psi(u)=f, \quad f \in Y, \\
B u=\widehat{A}(A u-\mathbf{z} P F(A u))-q \Psi(u)=f, \\
A u-z P F(A u)-\widehat{A}^{-1} q \Psi(u)=\widehat{A}^{-1} f, \\
F(A u)-F(\mathbf{z}) P F(A u)-F\left(\widehat{A}^{-1} q\right) \Psi(u)=F\left(\widehat{A}^{-1} f\right), \\
{\left[I_{n}-F(\mathbf{z}) P\right] F(A u)-F\left(\widehat{A}^{-1} q\right) \Psi(u)=F\left(\widehat{A}^{-1} f\right) .}
\end{gathered}
$$

From (12), since $u-\mathbf{z} N F(A u) \in D(\widehat{A})$ and $\widehat{A}$ is correct, we get

$$
\begin{gathered}
A(u-\mathbf{z} N F(A u))-\mathbf{z} P F(A u)-\widehat{A}^{-1} q \Psi(u)=\widehat{A}^{-1} f, \\
\widehat{A}(u-\mathbf{z} N F(A u))-\mathbf{z} P F(A u)-\widehat{A}^{-1} q \Psi(u)=\widehat{A}^{-1} f, \\
u-\mathbf{z} N F(A u)-\widehat{A}^{-1} \mathbf{z} P F(A u)-\widehat{A}^{-2} q \Psi(u)=\widehat{A}^{-2} f, \\
u-\left(\widehat{A}^{-1} \mathbf{z} P+\mathbf{z} N\right) F(A u)-\widehat{A}^{-2} q \Psi(u)=\widehat{A}^{-2} f, \\
\Psi(u)-\Psi\left(\widehat{A}^{-1} \mathbf{z} P+\mathbf{z} N\right) F(A u)-\Psi\left(\widehat{A}^{-2} q\right) \Psi(u)=\Psi\left(\widehat{A}^{-2} f\right), \\
{\left[I_{l}-\Psi\left(\widehat{A}^{-2} q\right)\right] \Psi(u)-\Psi\left(\widehat{A}^{-1} \mathbf{z} P+\mathbf{z} N\right) F(A u)=\Psi\left(\widehat{A}^{-2} f\right),}
\end{gathered}
$$

From (13), (15) we have

$$
\left(\begin{array}{cc}
I_{l}-\Psi\left(\widehat{A}^{-2} q\right) & -\Psi\left(\widehat{A}^{-1} \mathbf{z} P+\mathbf{z} N\right) \\
-F\left(\widehat{A}^{-1} q\right) & I_{n}-F(\mathbf{z}) P
\end{array}\right)\left(\begin{array}{c}
\Psi(u) \\
F(A u)
\end{array}\right)=\left(\begin{array}{c}
\Psi\left(\widehat{A}^{-2} f\right) \\
F\left(\widehat{A}^{-1} f\right)
\end{array}\right) .
$$

Let $\operatorname{det} L \neq 0$ and $u \in \operatorname{ker} B$. Then in (8) $f=0$ and

$$
B u=A^{2} u-g \Psi(u)=0, \quad \Phi(u)=N F(A u), \quad \Phi(A u)=P F(A u) .
$$

By the similar way as above is proved the type (16) for $f=0$, viz.

$$
\left(\begin{array}{cc}
I_{l}-\Psi\left(\widehat{A}^{-2} q\right) & -\Psi\left(\widehat{A}^{-1} \mathbf{z} P+\mathbf{z} N\right) \\
-F\left(\widehat{A}^{-1} q\right) & I_{n}-F(\mathbf{z}) P
\end{array}\right)\left(\begin{array}{c}
\Psi(u) \\
F(A u)
\end{array}\right)=\left(\begin{array}{c}
\overrightarrow{0} \\
\overrightarrow{0}
\end{array}\right),
$$

which, since $\operatorname{det} L \neq 0$ implies $\Psi(u)=\overrightarrow{0}, \quad F(A u)=\overrightarrow{0}$. Substituting these values into (17), we obtain $B u=A^{2} u=0, \Phi(u)=\Phi(A u)=\overrightarrow{0}$. Then $u \in D\left(\widehat{A}^{2}\right), \quad B u=\widehat{A}^{2} u=0$. The last implies $u=0$, since $\widehat{A}$ is correct. This proves that $\operatorname{ker} B=\{0\}$. So $B$ is injective.

Conversely. We will prove that if $B$ is injective, then $\operatorname{det} L \neq 0$, or equivalently if $\operatorname{det} L=0$, then $B$ is not injective. Let $\operatorname{det} L=0$. Then there exists a vector $\vec{c}=\operatorname{col}\left(\vec{c}_{1}, \vec{c}_{2}\right) \neq \overrightarrow{0}$,

$\vec{c}_{1}=\operatorname{col}\left(c_{11}, \ldots, c_{1 l}\right), \quad \vec{c}_{2}=\operatorname{col}\left(c_{21}, \ldots, c_{2 m}\right)$ such that $L \vec{c}=0$ or

$$
\left(\begin{array}{cc}
I_{l}-\Psi\left(\widehat{A}^{-2} q\right) & -\Psi\left(\widehat{A}^{-1} \mathbf{z} P+\mathbf{z} N\right) \\
-F\left(\widehat{A}^{-1} q\right) & I_{n}-F(\mathbf{z}) P
\end{array}\right)\left(\begin{array}{c}
\vec{c}_{1} \\
\vec{c}_{2}
\end{array}\right)=\left(\begin{array}{c}
\overrightarrow{0} \\
\overrightarrow{0}
\end{array}\right) .
$$

Consider the element $u_{0}=\widehat{A}^{-2} q \vec{c}_{1}+\left(\widehat{A}^{-1} \mathbf{z} P+\mathbf{z} N\right) \vec{c}_{2}$. It easy to verify that $u_{0} \neq 0$, otherwise since the components of $q$ are linearly independent, we get $\vec{c}_{1}=\overrightarrow{0}, \quad\left(\widehat{A}^{-1} \mathbf{z} P+\mathbf{z} N\right) \vec{c}_{2}=\overrightarrow{0}, \quad \mathbf{z} P \vec{c}_{2}=\overrightarrow{0}, \quad F(\mathbf{z}) P \vec{c}_{2}=\overrightarrow{0}$ and from (18) it follows that $\vec{c}_{2}=\overrightarrow{0}$. Thus $\vec{c}=\overrightarrow{0}$, which contradicts the hypothesis that $\vec{c} \neq \overrightarrow{0}$. Note that $u_{0} \in D(B)$, since $\Phi\left(u_{0}\right)=N \vec{c}_{2}, \quad \Phi\left(A u_{0}\right)=P \vec{c}_{2}, \quad F\left(A u_{0}\right)=F\left(\widehat{A}^{-1} q\right) \vec{c}_{1}+F(\mathbf{z}) P \vec{c}_{2} \quad$ and

$$
\begin{gathered}
\Phi\left(u_{0}\right)-N F\left(A u_{0}\right)=N \vec{c}_{2}-N F\left(\widehat{A}^{-1} q\right) \vec{c}_{1}-N F(\mathbf{z}) P \vec{c}_{2}= \\
=N\left(-F\left(\widehat{A}^{-1} q\right), I_{n}-F(\mathbf{z}) P\right) \operatorname{col}\left(\vec{c}_{1}, \vec{c}_{2}\right)=\overrightarrow{0},
\end{gathered}
$$




$$
\begin{gathered}
\Phi\left(A u_{0}\right)-P F\left(A u_{0}\right)=P \vec{c}_{2}-P F\left(\widehat{A}^{-1} q\right) \vec{c}_{1}-P F(\mathbf{z}) P \vec{c}_{2}, \\
=P\left(-F\left(\widehat{A}^{-1} q\right), I_{n}-F(\mathbf{z}) P\right) \operatorname{col}\left(\vec{c}_{1}, \vec{c}_{2}\right)=\overrightarrow{0} .
\end{gathered}
$$

The last equations in (19), (20) follow since (18). So $u_{0} \in D(B)$. We will show now that $u_{0} \in \operatorname{ker} B$.

$$
\begin{gathered}
B u_{0}=A^{2} u_{0}-q \Psi\left(u_{0}\right)=q \vec{c}_{1}-q \Psi\left(\widehat{A}^{-2} q\right) \vec{c}_{1}-q \Psi\left(\widehat{A}^{-1} \mathbf{z} P+\mathbf{z} N\right) \vec{c}_{2} \\
=q\left(I_{l}-\Psi\left(\widehat{A}^{-2} q\right),-\Psi\left(\widehat{A}^{-1} \mathbf{z} P+\mathbf{z} N\right)\right) \operatorname{col}\left(\vec{c}_{1}, \vec{c}_{2}\right)=q \overrightarrow{0}=\overrightarrow{0},
\end{gathered}
$$

since (18). So we obtain $u_{0} \neq \mathbf{0}$ and $u_{0} \in \operatorname{ker} B$. Hence $\operatorname{ker} B \neq\{0\}$ and $B$ is not injective. The statement (i) holds.

(ii) Let $\operatorname{det} L \neq 0$ and $B u=f$. From (8) as in the proof (i) we get (13), (14) and (15). Then

$$
\left(\begin{array}{c}
\Psi(u) \\
F(A u)
\end{array}\right)=\left(\begin{array}{cc}
I_{l}-\Psi\left(\widehat{A}^{-2} q\right) & -\Psi\left(\widehat{A}^{-1} \mathbf{z} P+\mathbf{z} N\right) \\
-F\left(\widehat{A}^{-1} q\right) & I_{n}-F(\mathbf{z}) P
\end{array}\right)^{-1}\left(\begin{array}{c}
\Psi\left(\widehat{A}^{-2} f\right) \\
F\left(\widehat{A}^{-1} f\right)
\end{array}\right)
$$

Substituting these values into (14) we obtain the solution (10) of the problem (8) for every $f \in Y$. Because $f$ in (10) is arbitrary, we obtain $R(B)=Y$. Since the operator $\widehat{A}^{-1}$ and the functionals $\Phi_{1}, \ldots, \Phi_{m}, \Psi_{1}, \ldots, \Psi_{l}$ are bounded, from (10) follows the boundedness of $B^{-1}$. Hence, the operator $B$ is correct if and only if (9) holds and the unique solution of (8) is given by (10). The theorem is proved.

From the previous theorem for $q=\overrightarrow{0}$ follows the next corollary which is useful for solving differential equations with integral boundary conditions.

Corollary 4. Let the spaces $X, Y$, the operators $A, \widehat{A}$, the vector $\mathbf{z}=\left(z_{1}, \ldots, z_{m}\right)$, functional vectors $\Phi, F$ and the matrices $N, P$ be defined as in Theorem 3 . Then:

(i) The operator $B$ defined by

$$
\begin{gathered}
B u=A^{2} u=f, \quad f \in Y, \\
D(B)=\left\{u \in D\left(A^{2}\right): \Phi(u)=N F(A u), \quad \Phi(A u)=P F(A u)\right\}
\end{gathered}
$$

is injective if and only if

$$
\operatorname{det} V=\operatorname{det}\left[I_{n}-F(\mathbf{z}) P\right] \neq 0 .
$$

(ii) If $B$ is injective, then $B$ is correct and for all $f \in Y$ the unique solution of (21) is given by

$$
u=B^{-1} f=\widehat{A}^{-2} f+\left(\widehat{A}^{-1} \mathbf{z} P+\mathbf{z} N\right) V^{-1} F\left(\widehat{A}^{-1} f\right) .
$$

Proof (i). For $q=0$ from (9) follows that

$$
\operatorname{det} L=\operatorname{det}\left(\begin{array}{cc}
I_{l} & -\Psi\left(\widehat{A}^{-1} \mathbf{z} P+\mathbf{z} N\right) \\
\mathbf{0}_{n l} & I_{n}-F(\mathbf{z}) P
\end{array}\right)=\operatorname{det}\left[I_{n}-F(\mathbf{z}) P\right]=\operatorname{det} V \neq 0 .
$$

It is easy to verify that

$$
L^{-1}=\left(\begin{array}{cc}
I_{l} & \Psi\left(\widehat{A}^{-1} \mathbf{z} P+\mathbf{z} N\right) V^{-1} \\
\mathbf{0}_{n l} & V^{-1}
\end{array}\right) .
$$

Then from (10) for $q=\overrightarrow{0}$ follows (22). $C[0,1]$

Example. The next problem with loaded differential equation and nonlocal integral boundary conditions on

$$
\begin{aligned}
& u^{\prime \prime}(t)-4 t u(1 / 2)-(2 t+1) u(1)=1-5 t, \\
& u(0)=-6 \int_{0}^{1} x^{2} u^{\prime}(x) d x+15 \int_{0}^{1} x^{4} u^{\prime}(x) d x, \\
& u^{\prime}(0)=6 \int_{0}^{1} x^{2} u^{\prime}(x) d x-15 \int_{0}^{1} x^{4} u^{\prime}(x) d x,
\end{aligned}
$$

is correct and the unique solution of (23) is given by

$$
u(t)=t^{2}-t+1
$$


Proof. First we rewrite the boundary conditions (24) in the form

$$
\begin{gathered}
u(0)=(-6,15) \operatorname{col}\left(\int_{0}^{1} x^{2} u^{\prime}(x) d x, \int_{0}^{1} x^{4} u^{\prime}(x) d x\right), \\
u^{\prime}(0)=(6,-15) \operatorname{col}\left(\int_{0}^{1} x^{2} u^{\prime}(x) d x, \int_{0}^{1} x^{4} u^{\prime}(x) d x\right) .
\end{gathered}
$$

If we compare (23), (25) with (8), it is natural to take $X=Y=C[0,1], \quad m=1, n=l=2, A u(t)=u^{\prime}(t)$, $D(A)=\left\{u(t) \in C^{1}[0,1]\right\}, X_{A}^{1}=C^{1}[0,1] . \quad A^{2} u=u^{\prime \prime}(t), D\left(A^{2}\right)=\left\{u(t) \in C^{2}[0,1]\right\}$. It is evident that $z=1$ constitute a basis of ker $A$. As the operator $\widehat{A}$ it is natural to take $\widehat{A} u(t)=A u(t)=u^{\prime}(t), D(\widehat{A})=\{u(t) \in$ $\in D(A): u(0)=0\}, \quad \widehat{A}^{2} u(t)=A^{2} u(t)=u^{\prime \prime}(t), D\left(\widehat{A}^{2}\right)=\left\{u(t) \in D\left(A^{2}\right): u(0)=u^{\prime}(0)=0\right\}, \quad$ The initial problem $\widehat{A} u(t)=f(t)$ is correct and has the unique solution $\widehat{A}^{-1} f(t)=\int_{0}^{t} f(x) d x$. Then, since (6), $\widehat{A}^{-2} f(t)=\int_{0}^{t}(t-x) f(x) d x$. By comparing (23), (25) with (8), it is natural to take $q_{1}=4 t, q_{2}=2 t+1$, $q=\left(q_{1}, q_{2}\right)=(4 t, 2 t+1), \quad f=1-5 t, \quad M=(-6,15), \quad P=(6,-15), \Phi(u)=u(0), \Phi(A u)=u^{\prime}(0)$,

$$
\Psi(u)=\left(\begin{array}{c}
\Psi_{1}(u) \\
\Psi_{2}(u)
\end{array}\right)=\left(\begin{array}{c}
u(1 / 2) \\
u(1)
\end{array}\right), \quad F(A u)=\left(\begin{array}{c}
F_{1}(A u) \\
F_{2}(A u)
\end{array}\right)=\left(\begin{array}{c}
\int_{0}^{1} x^{2} u^{\prime}(x) d x \\
\int_{0}^{1} x^{4} u^{\prime}(x) d x
\end{array}\right) .
$$

Then $F(f)=\left(\begin{array}{c}F_{1}(f) \\ F_{2}(f)\end{array}\right)=\left(\begin{array}{l}\int_{0}^{1} x^{2} f(x) d x \\ \int_{0}^{1} x^{4} f(x) d x\end{array}\right)$.

It is evident that $\Phi(z)=1$ for $z=1$ and that $\left|F_{1}(f)\right| \leq\|f\|_{C}, \quad\left|F_{2}(f)\right| \leq\|f\|_{C}$ for all $f \in C[0,1]$. So $F_{1}, F_{2} \in C^{*}[0,1]=Y^{*}$. Because of $|\Psi(u)|=\left|u\left(t_{0}\right)\right| \leq\|u(t)\|_{C} \leq\|u(t)\|_{C}+\left\|u^{\prime}(t)\right\|\left\|_{C}=\right\| u(t)\left\|_{C^{1}}=\right\| u(t) \|_{X_{A}^{1}}$, we conclude that $\Psi_{1}, \Psi_{2} \in\left[X_{A}^{1}\right]^{*}$. In the same way is proved that $\Phi \in\left[X_{A}^{1}\right]^{*}$. So we can apply Theorem 3 . We calculate $\widehat{A}^{-1} z=\int_{0}^{t} 1 d x=t, \quad \widehat{A}^{-1} z P+z N=t(6,-15)+(-6,15)=(6 t-6,-15 t+15)=\left(v_{1}, v_{2}\right)$,

$$
\begin{aligned}
& \Psi\left(\widehat{A}^{-1} z P+z N\right)=\left(\begin{array}{ll}
\Psi_{1}\left(v_{1}\right) & \Psi_{1}\left(v_{2}\right) \\
\Psi_{2}\left(v_{1}\right) & \Psi_{2}\left(v_{2}\right)
\end{array}\right)=\left(\begin{array}{cc}
v_{1}(1 / 2) & v_{2}(1 / 2) \\
v_{1}(1) & v_{2}(1)
\end{array}\right)=\left(\begin{array}{cc}
-3 & 15 / 2 \\
0 & 0
\end{array}\right), \\
& \widehat{A}^{-1} q_{1}(t)=\int_{0}^{t} 4 x d x=2 t^{2}, \quad \widehat{A}^{-1} q_{2}(t)=\int_{0}^{t}(2 x+1) d x=t^{2}+t, \\
& \widehat{A}^{-1} q=\left(2 t^{2}, t^{2}+t\right), \quad \widehat{A}^{-2} q_{1}(t)=\int_{0}^{t}(t-x) 4 x d x=\frac{2}{3} t^{3}, \\
& \widehat{A}^{-2} q_{2}(t)=\int_{0}^{t}(t-x)(2 x+1) d x=\frac{1}{3} t^{3}+\frac{1}{2} t^{2}, \\
& \widehat{A}^{-2} q=\left(\widehat{A}^{-2} q_{1}, \widehat{A}^{-2} q_{2}\right)=\left(\frac{2}{3} t^{3}, \frac{1}{3} t^{3}+\frac{1}{2} t^{2}\right) \\
& \Psi\left(\widehat{A}^{-2} q\right)=\left(\begin{array}{cc}
\Psi_{1}\left(\widehat{A}^{-2} q_{1}\right) & \Psi_{1}\left(\widehat{A}^{-2} q_{2}\right) \\
\Psi_{2}\left(\widehat{A}^{-2} q_{1}\right) & \Psi_{2}\left(\widehat{A}^{-2} q_{2}\right)
\end{array}\right)=\left(\begin{array}{cc}
\left(\widehat{A}^{-2} q_{1}\right)(1 / 2) & \left(\widehat{A}^{-2} q_{2}\right)(1 / 2) \\
\left(\widehat{A}^{-2} q_{1}\right)(1) & \left(\widehat{A}^{-2} q_{2}\right)(1)
\end{array}\right)= \\
& =\left(\begin{array}{cc}
1 / 12 & 1 / 6 \\
2 / 3 & 5 / 6
\end{array}\right) \\
& F\left(\widehat{A}^{-1} q\right)=\left(\begin{array}{cc}
F_{1}\left(\widehat{A}^{-1} q_{1}\right) & F_{1}\left(\widehat{A}^{-1} q_{2}\right) \\
F_{2}\left(\widehat{A}^{-1} q_{1}\right) & F_{2}\left(\widehat{A}^{-1} q_{2}\right)
\end{array}\right)=\left(\begin{array}{cc}
2 / 5 & 9 / 20 \\
2 / 7 & 13 / 42
\end{array}\right), \\
& I_{l}-\Psi\left(\widehat{A}^{-2} q\right)=\left(\begin{array}{cc}
11 / 12 & -1 / 6 \\
-2 / 3 & 1 / 6
\end{array}\right), \quad F(z)=\left(\begin{array}{c}
F_{1}(z) \\
F_{2}(z)
\end{array}\right)=\left(\begin{array}{l}
1 / 3 \\
1 / 5
\end{array}\right) \\
& F(z) P=\left(\begin{array}{l}
1 / 3 \\
1 / 5
\end{array}\right)(6,-15)=\left(\begin{array}{cc}
2 & -5 \\
6 / 5 & -3
\end{array}\right), \quad I_{n}-F(z) P=\left(\begin{array}{cc}
-1 & 5 \\
-6 / 5 & 4
\end{array}\right) \text {. }
\end{aligned}
$$


Then by using (9) we find

$$
L=\left(\begin{array}{cccc}
11 / 12 & -1 / 6 & 3 & -15 / 2 \\
-2 / 3 & 1 / 6 & 0 & 0 \\
-2 / 5 & -9 / 20 & -1 & 5 \\
-2 / 7 & -13 / 42 & -6 / 5 & 4
\end{array}\right)
$$

Since $\operatorname{det} L \neq 0$, by Theorem 3, the problem (23), is correct. We find the inverse matrix

$$
L^{-1}=\left(\begin{array}{cccc}
-140 / 303 & -548 / 303 & 70 / 101 & 175 / 101 \\
-560 / 303 & -374 / 303 & 280 / 101 & -700 / 101 \\
-248 / 909 & -565 / 1818 & 730 / 303 & -2135 / 606 \\
-1172 / 4545 & -289 / 909 & 299 / 303 & -889 / 606
\end{array}\right)
$$

For $f(t)=1-5 t$ we compute

$\widehat{A}^{-1} f(t)=\int_{0}^{t}(1-5 x) d x=t-\frac{5}{2} t^{2}, \quad \widehat{A}^{-2} f(t)=\int_{0}^{t}(t-x)(1-5 x) d x=\frac{1}{2} t^{2}-\frac{5}{6} t^{3}$,

$F_{1}\left(\widehat{A}^{-1} f\right)=\int_{0}^{1} x^{2}\left(x-\frac{5}{2} x^{2}\right) d x=-1 / 4, \quad F_{2}\left(\widehat{A}^{-1} f\right)=\int_{0}^{1} x^{4}\left(x-\frac{5}{2} x^{2}\right) d x=-4 / 21$,

$F\left(\widehat{A}^{-1} f\right)=\left(\begin{array}{c}-1 / 4 \\ -4 / 21\end{array}\right), \quad \Psi\left(\widehat{A}^{-2} f\right)=\left(\begin{array}{c}\Psi_{1}\left(\widehat{A}^{-2} f\right) \\ \Psi_{2}\left(\widehat{A}^{-2} f\right)\end{array}\right)=\left(\begin{array}{c}1 / 48 \\ -1 / 3\end{array}\right)$.

By substituting these values into (10) we obtain the unique solution of (23)

$$
u(t)=\frac{1}{2} t^{2}-\frac{5}{6} t^{3}+\left(\frac{2}{3} t^{3}, \frac{1}{3} t^{3}+\frac{1}{2} t^{2}, 6 t-6,-15 t+15\right) L^{-1}\left(\begin{array}{c}
1 / 48 \\
-1 / 3 \\
-1 / 4 \\
-4 / 21
\end{array}\right)
$$

which yields (24).

\section{References}

1 Нахушев А.М. Краевые задачи для нагруженных параболических уравнений и их применение к прогнозу уровня грунтовых вод / А.М. Нахушев, В.Н. Борисов // Дифференциальные уравнения. - 1977. - Вып. - 13. - № 1. - С. 105-110.

2 Нахушев А.М. Нагруженные уравнения и их приложения / А.М. Нахушев. - М.: Наука, 2012.

3 Нахушев А.М. Уравнения математической биологии / А.М. Нахушев. - М.: Высш. шк., 1995. $205 \mathrm{c}$.

4 Нахушев А.М. Нагруженные уравнения и их приложения / А.М. Нахушев // Дифференциальные уравнения. - 1983. - Вып. 19 (1). - С. 86-94.

5 Дженалиев М.Т. Нагруженные уравнения как возмущения дифференциальных уравнений / М.Т. Дженалиев, М.И. Рамазанов. - Алматы: Ғылым, 2010. - 334 с.

6 Владимиров В.С. Математические задачи теории переноса односкоростной теории переноса частиц / В.С. Владимиров // Тр. Мат. ин-та им. В.А. Стеклова АН СССР. - 1961. - 61. - 158 с.

7 Krall A.M. The development of general differential and general differential-boundary systems / A.M. Krall // Rocky Mountain J. Math. - 1975. - 5. - No. 4. - P. 493-542. https: // doi.org /10.1216 /RMJ-19755-4-493.

8 Lomov I.S. Loaded differential operators: Convergence of spectral expansions / I.S. Lomov // Diff. Equat. - 2014. - 50. - No. 8. - P. 1070-1079 https: // doi.org /10.1134 /S0012266114080060.

9 Lomov I.S. Study of Spectral Properties of a Loaded Second-Order Differential Operator / I.S. Lomov, V.V. Chernov // Diff. Equat. - 2015. - Vol. 51. - No. 7. - P. 857-861.

10 Дженалиев M.T. О граничной задаче для спектрально нагруженного оператора теплопроводности / М.Т. Дженалиев, М.И. Рамазанов // Сиб. мат. журн. - 2006. - Вып. 47. - № 3. - С. 527-547.

11 Дженалиев М.Т. Оптимальное управление линейными нагруженными параболическими уравнениями / М.Т. Дженалиев // Дифференциальные уравнения. - 1989. - 25. - No. 4. - С. 641-651. 
12 Ramazanov M.I. Boundary value problems for essentialy loaded parabolic equation / M.I. Ramazanov, M.T. Kosmakova, V.G. Romanovsky, B.H. Zhanbusinova, Zh.M. Tuleutaeva // Bulletin of the Karaganda University. Mathematics Series. - 2018. - Vol. 4. - No. 92. - P. 79-86.

13 Imanbaev N. To the eigenvalue problems of a special-loaded first-order differential operator / N. Imanbaev, B. Kalimbetov, Z. Khabibullayev // Int. J. Math. Anal. (Ruse). - 2014. - Vol. 8. - No. 45. - P. 22472254. http: // dx.doi.org /10.12988 /ijma.2014.48263.

14 Кожанов А.И. Нелинейные нагруженные уравнения и обратные задачи / А.И. Кожанов // Журн. вычислительной математики и мат физики. - 2004. - 44. - № 4. - С. 657-675.

15 Parasidis I.N. An exact solution method for a class of nonlinear loaded difference equa-tions with multipoint boundary conditions / I.N. Parasidis, E. Providas // Journal of Difference Equations and Applications, Taylor - Francis groop, Published on line. - 2018. - No. 05. - P. 1-15.

16 Dzhumabaev D. Computational methods for solving the boundary value problems for the loaded differential and Fredholm integro-differential equations / D. Dzhumabaev / Math. Meth. Appl. Sci. - 2017. P. 1-24. Available at https: // doi.org /10.1002/mma.4674.

17 Abdullaev V.M. Numerical method of solution to loaded nonlocal boundary value problems for ordinary differential equations / V.M. Abdullaev, K.R. Aida-Zade // Journal of Computational Mathematics and Mathematical Physics, Springer. - 2014. - Vol. 54. - No. 7. - P. 1096-1109.

18 Aida-Zade K.R. On the numerical solution of loaded system of ordinary differential equations with nonseparated multipoint and integral conditions / K.R. Aida-Zade, V.M. Abdullaev // Numerical Analysis and Applications, Pleiades Publishing, Ltd., 2014. - Vol. 7. - No. 1. - P. 1-14.

19 Alikhanov A.A. Boundary value problems for certain classes of loaded differential equa-tions and solving them by finite difference methods / A.A. Alikhanov, A.M. Berezgov, M.X. Shkhanukov-Lafishev // Comput. Math. and Math. Phys. Pleiades Publishing, Ltd., 2008. - 48. - No. 9. - P. 1581-1590. https: // doi.org /10.1134/S096554250809008X.

20 Ойнаров Р.О. Корректно разрешимые расширения операторов с конечными дефектами в банаховом пространстве / Р.О. Ойнаров, И.Н. Парасиди // Изв. АН КазССР. - 1988. - No. 5. - С. 42-46.

21 Ойнаров Р.О. Гладкие расширения минимального оператора с конечным дефектом в банаховом пространстве / Р.О. Ойнаров, С.С. Сагинтаева // Изв. НАН РК. Сер. физ.-мат. - 1994. - № 5. C. $43-48$.

22 Крейн М.Г. Теория самосопряженных расширений полуограниченных операторов Эрмита и их приложения / М.Г. Крейн // Мат. сб. - 1947. - 20. - No. 3. - С. 431-495.

23 Neumann J.Von. Allgemeine Eigenwerttheorie Hermitescher Functional operatoren / J.Von Neumann / Math. Ann., Bd. - 1929-1930. - 102. - P. 49-131.

24 Кокебаев Б.К. О сужениях и расширениях операторов / Б.К. Кокебаев, М.О. Отелбаев, А.Н. Шыныбеков // Докл. АН СССР. - 1983. - Вып. 271. - № 6. - С. 1307-1310.

25 Parasidis A.I. Loaded Differential and Fredholm Integro-Differential Equations with nonlocal integral boundary conditions [Electronic resource] / I.N. Parasidis, E. Providas, V. Dafopoulos // Applied Math. and control science. - 2018. - No.3. - P. 31-50. - Access mode: vestnik.pstu.ru.

\section{И.Н. Парасидис}

\section{Локальды емес шеттік интегралдық шарттарымен жүктелген дифференциалдық теңдеулер класстары үшін кеңейту әдісі}

\footnotetext{
Мақалада локальды емес шеттік интегралдық шарттарымен жүктелген кәдімгі дифференциалдық теңдеулер класы абстрактілі операторлық теңдеу терминінде зерттелді

$$
B u=A^{2} u-q \Psi(u)=f, \quad f \in Y,
$$$$
D(B)=\left\{u \in D\left(A^{2}\right): \Phi(u)=N F(A u), \quad \Phi(A u)=P F(A u)\right\} .
$$

Бұл теңдеулердің жүктелген бөлігі және локальды емес шеттік интегралдық шарттары сәйкесінше $\Psi(u)$ және $F(A u)$, функционалдық векторларының көмегімен сипатталды. Мұндай теңдеулер сызықтық операторларды кеңейту теориясынан шығады. Қарастырылатын теңдеулердің шешілгіштігінің
} 
қажетті және жеткілікті шарттары қандай да бір матрицаның анықтауышы көмегімен өрнектеледі. Жүктелген теңдеулердің қарастырылатын класстарының нақты шешуін табу үшін, анықтауыш нольге тең емес жағдайында, дайын формула ұсынылады. Егер қандай да бір есеп (1) түріне келтірілетін болса, онда оны ұсынылып отырған кеңейту әдісімен оңай шешуге болады. Сонымен қоса, $q=\overrightarrow{0}$, үшін бұл әдіс шешілгіштіктің қажетті және жеткілікті шарттарын және локальды емес шеттік интегралдық шарттарымен жүктелген кәдімгі дифференциалдық теңдеулер кластарын абстрактілі операторлық теңдеу терминінде нақты шешу үшін формула береді.

Kiлm сөздер: жүктелген кәдімгі дифференциалдық теңдеулер, дифференциалдық теңдеулер, локальды емес интегралдық шеттік шарттар, инъективті және дұрыс операторлар, нақты шешулер.

\section{И.Н. Парасидис}

\section{Метод расширения для класса нагруженных дифференциальных уравнений с нелокальными граничными интегральными условиями}

В статье исследован класс нагруженных обыкновенных дифференциальных уравнений с нелокальными граничными интегральными условиями в терминах абстрактного операторного уравнения

$$
\begin{gathered}
B u=A^{2} u-q \Psi(u)=f, \quad f \in Y ; \\
D(B)=\left\{u \in D\left(A^{2}\right): \Phi(u)=N F(A u), \quad \Phi(A u)=P F(A u)\right\} .
\end{gathered}
$$

Нагруженная часть и нелокальные граничные интегральные условия этих уравнений описываются с помощью функциональных векторов $\Psi(u)$ и $F(A u)$ соответственно. Такие уравнения следуют из теории расширений линейных операторов. Необходимые и достаточные условия разрешимости рассматриваемых уравнений выражаются с помощью определителя некоторой матрицы. В случае когда этот определитель ненулевой, предлагается готовая формула для нахождения точного решения рассматриваемого класса нагруженных уравнений. Если некоторая задача может быть приведена к виду (1), то ее можно легко решить предлагаемым методом расширения. Данный метод для $q=\overrightarrow{0}$ также дает необходимые и достаточные условия разрешимости и формулу для точного решения для класса обыкновенных дифференциальных уравнений с нелокальными граничными интегральными условиями в терминах абстрактного операторного уравнения

$$
B u=A^{2} u=f ; \quad D(B)=\left\{u \in D\left(A^{2}\right): \Phi(u)=N F(A u) ; \quad \Phi(A u)=P F(A u)\right\} ; \quad f \in Y .
$$

Ключевые слова: нагруженные обыкновенные дифференциальные уравнения, дифференциальные уравнения, нелокальные интегральные граничные условия, инъективные и корректные операторы, точные решения.

\section{References}

1 Nakhushev, A.M., \& Borisov, V.N. (1977). Kraevye zadachi dlia nahruzhennykh parabolicheskikh uravnenii i ikh primenenie $\mathrm{k}$ prohnozu urovnia hruntovykh vod [Boundary value problems for loaded parabolic equations and their application to forecast of groundwater level]. Differentsialnye uravneniia - Differential equations, 13, 1, 105-110 [in Russian].

2 Nakhushev, A.M. (2012). Nahruzhennye uravneniia $i$ ikh prilozheniia /Loaded equations and their applications]. Moscow: Nauka [in Russian].

3 Nakhushev, A.M. (1995). Uravneniia matematicheskoi biolohii [Equations of Mathematical Biology]. Moscow: Vysshaia shkola [in Russian]

4 Nakhushev, A.M. (1983). Nahruzhennye uravneniia i ikh prilozheniia [Loaded equations and their applications]. Differentsialnye uravneniia - Differential equations, 19, 1, 86-94 [in Russian].

5 Dzhenaliev, M.T., \& Ramazanov, M.I. (2010). Nahruzhennye uravneniia kak vozmushcheniia differentsialnykh uravnenii [Loaded equations as perturbations of differential Equations]. Almaty: Hylym [in Russian]. 
6 Vladimirov, V.S. (1961). Matematicheskie zadachi odnoskorostnoi teorii perenosa chastits [Mathematical problems in one-speed theory of particle transport]. Trudy Matematicheskoho instituta imeni V.A. Steklova Akademii nauk SSSR - Proceeding of V.A. Steklov Math. Inst., 61, 3-158 [in Russian].

7 Krall, A.M. (1975). The development of general differential and general differential-boundary systems Rocky Mountain J. Math., 5, 4, 493-542. Retrieved from https://doi.org/10.1216/RMJ-1975- 5-4-493.

8 Lomov, I.S. (2014). Loaded differential operators: Convergence of spectral expansions. Differential equations, 50, 8, 1070-1079. Retrieved from https://doi.org/10.1134/S0012266114080060.

9 Lomov, I.S., \& Chernov, V.V. (2015). Study of Spectral Properties of a Loaded Second-Order Differential Operator. Differential equations, 51, 7, 857-861.

10 Dzenaliev, M.T., \& Ramazanov, M.I. (2006). O hranichnoi zadache dlia spektralno-nahruzhennoho operatora teploprovodnosti [On the boundary value problem for the spectrally loaded heat condition operator]. Sibirskii matematicheskii zhurnal - Sibirian Math. Journal, 47, 3, 527-547 [in Russian].

11 Dzhenaliev, M.T. (1989). Optimalnoe upravlenie lineinymi nahhruzhennymi parabolicheskimi uravneniiami [Optimal control of linear loaded parabolic equations]. Differentsialnye uravneniia - Differential equations, 25, 4, 641-651 [in Russian].

12 Ramazanov, M.I., Kosmakova, M.T., Romanovsky, V.G., Zhanbusinova, B.H., \& Tuleutaeva, Zh.M. (2018). Boundary value problems for essentialy loaded parabolic equation. Bulletin of the Karaganda University. Mathematics Series, 4, 92, 79-86.

13 Imanbaev, N., Kalimbetov, B., \& Khabibullayev, Z. (2014). To the eigenvalue problems of a specialloaded first-order differential operator. Int. J. Math. Anal. (Ruse), 8, 45, 2247-2254. Retrieved from http://dx.doi.org/10.12988/ijma.2014.48263.

14 Kozhanov, A.I. (2004). Nelineinye nahruzhennye uravneniia i obratnye zadachi [Nonlinear Loaded Equations and Inverse Problems]. Zhurnal vychislitelnoi matematiki i matematicheskoi fiziki - J. Comput. Math. Math. Phys, 44. 4, 657-675 [in Russian].

15 Parasidis, I.N., \& Providas, E. (2018). An exact solution method for a class of nonlinear loaded difference equations with multipoint boundary conditions. Journal of Difference Equations and Applications, Taylor - Francis groop, Published on line, 05, 1-15.

16 Dzhumabaev, D. (2017). Computational methods for solving the boundary value problems for the loaded differential and Fredholm integro-differential equations. Math. Meth. Appl. Sci., 1-24. Retrieved from https://doi.org/10.1002/mma.4674.

17 Abdullaev, V.M., Aida-Zade, K.R. (2014). Numerical method of solution to loaded nonlocal boundary value problems for ordinary differential equations. Journal of Computational Mathematics and Mathematical Physics, Springer, 54, 7, 1096-1109.

18 Aida-Zade, K.R., \& Abdullaev, V.M. (2014). On the numerical solution of loaded system of ordinary differential equations with nonseparated multipoint and integral conditions. Numerical Analysis and Applications, Pleiades Publishing, LTD., 7, 1, 1-14.

19 Alikhanov, A.A., Berezgov, A.M., \& Shkhanukov-Lafishev, M.X. (2008). Boundary value problems for certain classes of loaded differential equations and solving them by finite difference methods. Comput. Math. and Math. Phys., Pleiades Publishing, LTD., 48, 9, 1581-1590. https://doi.org/10.1134/S09655425 0809008X.

20 Oinarov, R.O., \& Parasidi, I.N. (1988). Korrektno razreshimye rasshireniia operatorov s konechnymi defektami v banakhovom prostranstve [Correct extensions of operators with finite defect in Banach spases]. Izvestiia AN KazSSR, 5, 42-46 [in Russian].

21 Oinarov, R.O., \& Sagintaeva, S.S. (1994). Hladkie rasshireniia minimalnoho operatora s konechnym defektom v banakhovom prostranstve [Smooth extensions of a minimal operator with a finite defect in a Banach space]. Izvestiia NAN RK. Seriia Fiz.-mat., 5, 43-48 [in Russian].

22 Krein, M.G. (1947). Teoriia samosopriazhennykh rasshirenii poluohranichennykh operatorov Ermita i ikh prilozheniia [The theory of self-adjoint extensions of semi-bounded Hermitian operators and its aplications] Matematicheskii sbornik - Math. Notes, 20, 3, 431-495 [in Russian].

23 J.von Neumann. (1929-1930). Allgemeine Eigenwerttheorie Hermitescher Functional operatoren. Math. Ann., Bd., 102, 49-131. 
24 Kokebaev, B.K., Otelbaev, M., \& Shynybekov, A.N. (1983). O suzheniiakh i rasshireniiakh operatorov [About Restrictions and Extensions of operators]. Doklady AN SSSR - Reports of the USSR Academy of Sciences, 271, 6, 1307-1310 [in Russian].

25 Parasidis, I.N., Providas, E., \& Dafopoulos, V. (2018). Loaded Differential and Fredholm IntegroDifferential Equations with nonlocal integral boundary conditions. Applied Math. and control science, vestnik.pstu.ru., 3, 31-50. 